***Please note that this manuscript has not been peer-reviewed or published***

The Temporal Stability and Construct Validity of Measures Assessing

Mania Symptoms and Risk: A Comparative Analysis

\author{
Kasey Stanton \\ Virginia Tech, Western University \\ David Watson \\ University of Notre Dame
}

Correspondence concerning this paper should be addressed to Kasey Stanton at kaseyjstanton@gmail.com. There is no funding to report for this research, and informed consent was received from all study participants. This manuscript has not been peer-reviewed or published as noted above. 


\begin{abstract}
Fluctuations in mood and activity levels are defining features of bipolar disorder, but the temporal stability of measures used to assess symptoms and traits relevant to bipolar disorder is unclear. This study examined the short-term stability of several widely used, contemporary bipolar disorder measures (e.g., Altman Self-Rating Mania Scale, General Behavior Inventory, Hypomanic Personality Scale, Mood Disorder Questionnaire) over a period of roughly 2 weeks ( $M$ Retest Interval $=15.17$ days) in an undergraduate sample. The stability correlations varied widely, ranging from .49 to .83 . As would be expected, measures that were designed to assess traits related to bipolar disorder tended to show stronger stability than scales purporting to assess more transient symptoms of bipolar disorder. Other analyses revealed that — consistent with previous researchsome bipolar disorder scales demonstrated moderate to strong positive relations with neuroticism/negative affect and other psychopathology, whereas others related weakly to such measures but showed more robust positive relations with extraversion/positive affect. Taken together, our findings suggest that it is important to consider administration instructions (e.g., trait vs. symptom ratings), subscale properties, and item format when selecting study measures in bipolar disorder research.
\end{abstract}


The Temporal Stability and Construct Validity of Measures Assessing Mania Symptoms and Risk: A Comparative Analysis

Mania is the core criterion for bipolar disorder (BD), a psychiatric illness centrally characterized by emotional disturbance (American Psychiatric Association [APA], 2013). Measures such as the Altman Self-Rating Mania Scale (ASRM; Altman, Hedeker, Peterson, \& Davis, 1997), General Behavior Inventory (GBI; Depue, Krauss, Spoont, \& Arbisi 1989), Hypomanic Personality Scale (HPS; Eckblad \& Chapman, 1986), and Mood Disorder Questionnaire (MDQ; Hirschfeld et al., 2000) have been widely used in BD research. However, less research has examined the psychometric properties of these measures compared to measures of other psychopathology such as depression (Miller, Johnson, \& Eisner, 2009), and little research has directly evaluated the degree to which these measures show convergence, even though they vary widely in their content, format, and administration instructions (Stanton, Gruber, \& Watson, 2017).

The ASRM assesses current manic symptoms, whereas the MDQ is a BD screening measure assessing the lifetime occurrence of manic symptoms. The HPS assesses a stable "hypomanic personality" thought to confer risk for manic episodes, which has been described as a gregarious, confident, hypersocial, and energetic personality style (Eckblad \& Chapman, 1986). Similarly, the GBI assesses mania risk, with Depue and colleagues (1989) describing it as a measure "developed to identify bipolar affective conditions on a lifetime or trait basis" (p. 117). For convenience, we refer to these measures from hereon as "bipolar-relevant" (BR), as they — with the exception of the ASRM — are intended to assess risk for mania and traits related to mania in both non-clinical and clinical samples rather than current manic symptoms per se. 
Compared to the literatures for many other forms of psychopathology, there has been relatively little investigation into the psychometric properties of these BR measures as stated, including a dearth of research examining the stability of scores assessed by these measures (Carpenter, Zimmerman, Emery, \& Stanton, 2020; Chmielewski \& Watson, 2009; Miller et al., 2009; Mohammadzadeh \& Monazzami, 2018; Stanton et al., 2019). This is surprising in some ways given that BD is defined by fluctuations in mood, energy, and activity levels (Sperry, Barrantes-Vidal, \& Kwapil, 2018). Scores from these BR measures might be thought to be inherently unstable given the central role of affective lability in BD; however, BR measures designed to assess traits and the lifetime occurrence of symptoms should show strong stability over relatively short intervals. For example, we would expect the HPS to yield strong test-retest correlations over a period of several weeks because no true change in a stable hypomanic personality would be expected over such an interval. In contrast, the ASRM targets short-term, transient symptoms over the past week. Consequently, we would expect it to yield weaker testretest coefficients than the HPS over short periods, given that change in transient symptoms could occur over this interval. Thus, our first study goal was to determine the stability of selfreport BR measures.

Given that BR measures vary in their content, format, and administration instructions, our second goal was to explicate their construct validity by examining both (a) their inter-relations, and (b) the degree to which they show similarities in their associations with other constructs theoretically related to BD (e.g., personality/affect, internalizing symptoms, positive urgency). The inclusion of personality, affect, and other psychopathology measures also provided "benchmarks" to aid in the interpretation of the test-retest correlations from our stability analyses (see Watson, 2004). These "benchmarks" permit one to conduct comparative tests of stability 
across constructs hypothesized to be more or less stable than the constructs of interest. For example, we would expect scales assessing personality traits—which show strong dependability over relatively short periods (Gnambs, 2014) — to yield higher test-retest correlations than BR measures assessing transient symptoms (i.e., the ASRM). In contrast, however, we would expect BR measures assessing traits related to BD (i.e., the HPS and GBI) to yield stability estimates similar to those for personality measures.

\section{Study Predictions}

We examined the temporal stability of BR measures over a period of approximately 2 weeks to address our first goal of determining their comparative stability. We chose this interval because it allowed us to compare the retest stability of measures over a time period in which we expected some measures to show strong test-retest correlations (i.e., trait measures such as the HPS and GBI) and others to show comparatively weaker test-retest correlations (i.e., symptom measures such as the ASRM).

Mania and BD are somewhat unique in that they relate positively to both positive affect/extraversion and negative affect/neuroticism (Watson \& Naragon-Gainey, 2010). In this regard, specific content within these BR measures may show differential relations to personality and affect. For example, Schalet, Durbin and Revelle (2011) found that the HPS items defined a three-factor structure of Social Vitality, Mood Volatility, and Excitement. Whereas Social Vitality and Excitement related positively to extraversion, Mood Volatility associated negatively with extraversion, but positively with neuroticism. These findings indicate distinctive properties for subscales within measures, and as a result, we examined the stability and correlates of subscales rather than for total scores when applicable. We expected to replicate the HPS subscale relations with personality found by Schalet et al. (2011), and we predicted the BR 
measures to show moderate to strong positive inter-relations given that all are intended to assess mania risk and/or symptoms. We examined other associations with personality, affect, and other psychopathology on an exploratory basis.

\section{Method}

\section{Participants}

The sample consisted of 382 undergraduates recruited from a psychology department research participant pool. Participants completed the study measures and were re-assessed using the same battery approximately 2 weeks later $(M$ interval $=15.17$ days $)$. Of the original sample, $291(76.2 \%)$ completed the Time 2 measures (however, sample sizes for specific analyses vary slightly due to missing data). The majority of the participants who completed both assessments identified as White or European American (70.1\% White/European American and 10.7\% AsianAmerican; remaining participants endorsed a range of other identities). Additionally, 9.3\% of sample participants identified as Hispanic or Latino/Latinx, and most participants identified as female (70.8\%). Participants reported a mean age of 19.18 years $(S D=1.16)$.

\section{Bipolar-Relevant Trait Measures}

General Behavior Inventory (GBI; Depue et al., 1989). The GBI assesses "symptomatic behaviors associated with depression and hypomania/mania" (Depue et al., 1989, p. 118) by asking participants if they have experienced a range of symptoms in their lifetimes. In addition to assessing mania and depression, the GBI also includes "biphasic" items, which describe the fluctuations between depression and mania in the same item. Participants completed the 28 biphasic (seven items) and hypomanic/manic (21 items) items, rating the frequency with which they have experienced symptoms using a 4-point scale ranging from never or hardly ever to very often or almost constantly. 
Hypomanic Personality Scale (HPS; Eckblad \& Chapman, 1986). As discussed, the HPS assesses a personality style thought to confer risk for mania, and we report results based on Schalet and colleagues' (2011) three-factor structural model (Social Vitality, 19 items; Mood Volatility, 13 items; Excitement, seven items). Participants responded to the items using a truefalse response format.

Mood Disorder Questionnaire (MDQ; Hirschfeld et al., 2000). The MDQ's 13 items ask participants if they have ever experienced a range of BD symptoms in their lifetimes. Participants responded to the items used a dichotomous "Yes" or "No" response format. The MDQ can be scored dimensionally (Carpenter et al., 2020; Stanton \& Watson, 2017), and we used this scoring method here.

Personality Inventory for DSM-5 (PID-5; Krueger, Derringer, Markon, Watson, \& Skodol, 2012). The PID-5 assesses personality pathology using items that describe long-term patterns of thought, behavior, and emotion. Only the PID-5 Emotional Lability scale (seven items; e.g., "unpredictable moods") was administered. Although it is not a measure of BD per se, it was included in our battery because it assesses a dimension related to BD. Participants responded using a 4-point scale ranging from Very False or Often False to Very True or Often True.

\section{Bipolar-Relevant Symptom Measures}

Altman Self-Rating Mania Scale (ASRM; Altman et al., 1997). The ASRM includes five groups of statements assessing symptoms over the past week. Its items assess levels of euphoric mood, increased self-esteem, decreased need for sleep, pressured speech, and psychomotor agitation. 


\section{Inventory of Depression and Anxiety Symptoms-II (IDAS-II; Watson et al., 2012).}

Participants completed the IDAS-II Euphoria (five items; e.g. "elated for no reason") and Mania (five items; "racing thoughts") scales, both of which assess manic symptoms. They also completed the Dysphoria (10 items; e.g., "felt depressed"), Lassitude (six items; e.g., “exhausted”), Insomnia (six items), Appetite Gain (three items), and Appetite Loss (three items) scales to assess depression. Participants responded using a 5-point scale ranging from not at all to extremely in regard to the past 2 weeks.

\section{Personality/Affectivity Measures}

Big Five Inventory (BFI; John \& Srivastava, 1999). The BFI assesses the five-factor model personality traits of neuroticism ( 8 items), extraversion ( 8 items), openness (10 items), agreeableness (9 items) and conscientiousness (9 items). Participants responded using a 5-point scale ranging from disagree strongly to agree strongly.

\section{Positive and Negative Affect Schedule (PANAS; Watson, Clark, \& Tellegen, 1988).} The PANAS assesses negative (10 items; e.g., "distressed") and positive affect (10 items; e.g., "enthusiastic"). Participants were given trait instructions asking them to indicate "to what extent you generally feel this way, that is, how you feel on average," and they rated themselves using a 5-point scale ranging from very slightly or not at all to extremely.

Positive Urgency Measure (PUM; Cyders et al., 2007). The PUM assesses the tendency to respond recklessly when experiencing strong positive mood states, which is described as characteristic of manic episodes (APA, 2013). Participants responded using a 4-point scale ranging from very much like me to not at all like me.

\section{Results}

\section{Overview}


Table 1 presents scale descriptive statistics at both assessments. Mean scores from Time 1 to Time 2 were significantly different in some cases; however, all differences were "small" in magnitude (Cohen, 1988). We first present inter-scale correlations among all BR scales at both assessments (Table 2). Next, we report the correlations between the BR scales and depression, positive urgency, affect, and personality (Table 3). Lastly, we present the test-retest stability coefficients for the BR scales (Table 4) and for the "benchmark" scales (Table 5).

\section{Bipolar-Relevant Inter-Correlations}

All BR scales correlated positively at both Times 1 and 2 (see Table 2), but some scales showed much stronger inter-scale relations than others. For example, HPS Mood Volatility and GBI Mania had moderate to strong associations with other BR scales in nearly all instances (both had at least five correlations $\geq .50$ at both Times 1 and 2$)$; in contrast, HPS Social Vitality ( $r=$ .51 with HPS Excitement at Time 2$)$ and the ASRM ( $r=.54$ with IDAS-II Euphoria at Time 2) each had only one association $\geq .50$ across assessments.

The inter-scale correlations do not seem to be dictated by whether a scale was designed to be a "symptom" or "trait” BR measure. For example, IDAS-II Mania (a symptom measure) correlated more strongly with GBI Mania (a trait measure; $r=.59$ and .65 at Times 1 and 2, respectively) than it did with the ASRM (another symptom measure; $r=.22$ and .30 at Times 1 and 2, respectively). Thus, these correlations were driven more by item content than by the assessed time frame.

\section{Bipolar-Relevant Correlations with Other Measures}

Correlations between the BR scales and other study scales at Time 1 are shown in Table 3; we focused on the Time 1 data because of the larger initial sample size. Five BR scales-HPS Mood Volatility, GBI Mania, GBI Biphasic, PID-5 Emotional Lability, and IDAS-II Mania- 
correlated strongly with both IDAS-II Dysphoria ( $r$ s ranged from .52 to .62 , mean $r=.57$ ) and PANAS Negative Affect ( $r$ s ranged from .50 to .57 , mean $r=.52$ ). These scales also tended to have moderate to strong associations with Positive Urgency ( $r$ s ranged from .36 to .52 , mean $r=$ .44 ), BFI Neuroticism ( $r$ s ranged from .25 to .63 , mean $r=.43$ ), and IDAS-II Lassitude ( $r$ s ranged from .39 to .49 , mean $r=.42$ ).

In contrast, the five other BR measures-HPS Social Vitality, HPS Excitement, the MDQ, IDAS-II Euphoria, and the ASRM-exhibited weaker associations. In fact, these scales jointly yielded only a single association $\geq|.40|$ ( $r=.60$ for HPS Social Vitality with extraversion).

\section{Bipolar-Relevant Scale Stability}

The test-retest correlations for each BR measure are depicted in Table 4. The coefficients varied widely, ranging from .83 (HPS Social Vitality) to .49 (ASRM). Trait measures such as the HPS and PID-5 produced higher test-retest coefficients than measures such as the IDAS-II and ASRM that assess symptoms over short periods. The seven trait-oriented measures had retest correlations ranging from .68 to .83 (mean $=.73$ ); in contrast, the three symptom measures had coefficients ranging from .49 to $.64($ mean $=.58)$.

There are several notable findings. First, although all three HPS subscales had retest correlations greater than .70 , the coefficient for the Social Vitality subscale (.83) was significantly higher than those for the Mood Volatility and Excitement subscales (.71 and .72, respectively). Consistent with this, the test-retest correlation for IDAS-II Mania (.64) was not significantly different from those for HPS Mood Volatility or Excitement. Relatedly, the MDQ (.68) produced a test-retest coefficient that was not significantly different from those yielded by either IDAS-II Mania (.64) or Euphoria (.60). The stability estimates for both GBI Biphasic 
(.72) and Mania (.70)—which assess traits related to BD—were significantly higher than those for IDAS-II Euphoria (.60), but not IDAS-II Mania (.64).

\section{Comparisons between Bipolar-Relevant and "Benchmark" Scales}

Examining the stability of other study scales used as "benchmarks" (see Table 5) provides additional information in interpreting the stability of the BR measures. With the exception of the HPS Social Vitality subscale and the ASRM, the test-retest correlations for most BR measures were similar in magnitude to the coefficients for measures of affect, positive urgency, and depression symptoms. Both GBI scales $(r s=.72$ and .70 for the Biphasic and Mania scales, respectively) and the MDQ (.68) were significantly less stable than all of the personality scales, despite the fact that these measures were designed to assess stable constructs.

Looking specifically at the HPS subscales, the test-retest correlation for Social Vitality was not significantly different from those for any of the BFI scales, with the exception of BFI Extraversion (.90). In contrast, the test-retest coefficients for Mood Volatility and Excitement were significantly weaker than those for all five BFI scales. The test-retest coefficient for Social Vitality also was significantly stronger than those for both PANAS Positive and Negative Affect (both $r \mathrm{~s}=.73$ ), positive urgency $(r=.67)$, and for all depression symptom measures ( $r$ s ranged from .56 to .74). Finally, the ASRM stability coefficient was significantly weaker than the testretest correlations for any of the other symptom domains assessed, with the exception of IDAS-II Appetite Loss (.56).

\section{Comparisons within the "Benchmark" Scales}

Several aspects of the Table 5 data are interesting in their own right. For example, consistent with previous research indicating that personality measures show comparatively stronger temporal stability than measure of trait affectivity (Chmielewski \& Watson, 2009; 
Watson, 2004), our data demonstrated that the test-retest coefficient for BFI Extraversion (.90) was significantly stronger than the coefficient for PANAS Positive Affect (.73) and that BFI Neuroticism (.85) produced a significantly higher test-retest correlation than PANAS Negative Affect (.73). More generally, it is noteworthy that all of the BFI scales demonstrated significantly stronger test-retest coefficients than any of the other scales in Table 5, with BFI Extraversion yielding a significantly stronger stability coefficient than any of the other BFI scales.

\section{Discussion}

The primary goals of this study were to (a) compare the temporal stability of BR measures, and (b) explicate the construct validity of such measures vis-à-vis their inter-relations and relations with personality, affect, and other psychopathology. Our results generally indicated that measures such as the HPS and PID-5 using "trait" instructions produced stronger test-retest reliability estimates over a period of roughly 2 weeks than "symptom" measures such as the IDAS-II scales and the ASRM. Although all of the BR scales correlated positively (see Table 2), some (i.e., HPS Mood Volatility and GBI Mania) displayed moderate to strong inter-relations, whereas others (i.e., HPS Social Vitality, the ARSM) demonstrated comparatively weaker interrelations. Furthermore, the BR scales displayed notable disparities in their associations with other variables (see Table 3). Thus, although these scales clearly overlap in their content, some also seem to tap content not well-captured by others. Next, we discuss particularly informative findings and the broader implications of our results.

\section{Broader Assessment Implications}

Our results bear important assessment-related implications. First, some measures purporting to assess BR traits and risk for mania displayed similar stability to BR symptom 
measures, even though we expected the former to show stronger stability. For example, both GBI scales and the MDQ_ — which all assess the lifetime occurrence of symptoms_-were only as stable as IDAS-II Mania, a current symptom measure. We expected the GBI scales to show stability comparable to that for personality traits, given that these scales were developed to assess long-term patterns of behavior thought to confer mania risk. However, both scales were significantly less stable than all Big Five personality traits, yielding test-rest coefficients similar to those for the affectivity scales. Researchers should take this information into account to ensure measures are used for their intended purposes; based on this evidence, some measures seem better suited to assess stable traits than others. The GBI scales and MDQ may have yielded weaker test-retest coefficients than predicted because they instruct participants to think retrospectively. This may encourage episodic processing, which is more biased than semantic memory (Robinson \& Clore, 2002). As a result, we encourage the use of administration formats (e.g., general behavior ratings) that ask participants to rate their generalized self-view and selfconcepts - in other words, formats that access semantic rather than episodic memory — whenever possible (Watson, 2004).

Our results also indicate that some BR scales seem to tap content related neuroticism and mood lability (e.g., HPS Mood Volatility, GBI Mania, GBI Biphasic), whereas others assess content associated with extraversion/positive affect (e.g., HPS Social Vitality and Excitement, ASRM). Our findings are consistent with those presented by Watson and Naragon-Gainey (2014), who reported results based on many of these same scales (i.e., the HPS, GBI, and IDASII). They found evidence that BR scales define two factors: one characterized by negative affectivity (marked by both GBI scales, HPS Mood Volatility, and IDAS-II Mania) and the other by elation (marked by HPS Social Vitality, HPS Excitement, and IDAS-II Euphoria). Other 
recent factor analytic studies have yielded similar results (e.g., Carpenter et al., 2020; also see Ruggero et al., 2014 for an overview of other studies examining the factor structures of BR measures). We urge researchers to consider these distinctions carefully when selecting BR measures, and it may be advisable to use several different BR measures within studies to ensure adequate coverage of both the negative affectivity and elation dimensions that define BD (Stanton, McDonnell, Hayden, \& Watson, 2020). For instance, based on our findings, we would expect research using the GBI—which appears to assess negative affectivity — to yield different results than research with the ASRM-which appears to tap elation.

Lastly, our data highlight the importance of considering the psychometric properties of subscales within measures. For example, the HPS Social Vitality subscale demonstrated the strongest test-retest coefficient of any BR measure, producing a reliability estimate comparable to those for personality measures. However, the HPS Mood Volatility and Excitement subscales were less stable, producing retest estimates similar to those for some symptom measures. Furthermore, the HPS subscales also showed different correlates, with Mood Volatility associating more strongly with other BR measures and neuroticism/negative affect. In contrast, HPS Social Vitality and Excitement showed weaker BR inter-relations, and the former related strongly to extraversion.

\section{Limitations, Future Directions, and Conclusion}

Strengths of this study include its administration of a number of BR measures concurrently, which permitted direct comparisons of their content overlap, patterns of correlates, and stability over an interval of roughly 2 weeks. Nevertheless, it had limitations worth noting. First, we used data from undergraduate participants who generally reported relatively low levels of BR symptoms; it therefore is unclear to what extent our findings would generalize to clinical 
samples. However, regarding this limitation, it should be noted that (1) many of the BR measures were constructed to assess mania risk and symptoms in non-clinical as well clinical samples (Sperry, Walsh, \& Kwapil, 2015), and (2) several BR scales (e.g., the HPS and GBI) were first validated in student samples (Depue et al., 1989; Eckblad \& Chapman, 1986). Next, our sample size was larger than that for many previous studies examining the stability of psychopathology measures, but was still slightly under the target size of 300-400 participants recommended for retest studies by Watson (2004). Finally, our study included only one retest interval of roughly 2 weeks. Studies including additional timepoints would be useful to build from this work, including studies adopting intensive longitudinal designs examining the extent to which BR measures predict patterns of mood and symptom fluctuation in daily life (e.g., Sperry et al., 2018).

These limitations aside, we believe that our data illustrate that BR measures differ meaningfully in their item content, correlates, and stability over relatively short retest intervals. Therefore, our findings suggest that it is important to consider administration instructions, item content, and subscale properties when selecting BR measures. Future research should assess the stability and construct validity of BR measures in clinical samples, and research incorporating more intensive longitudinal assessment approaches would prove useful as noted. 


\section{References}

Altman, E. G., Hedeker, D., Peterson, J. L., \& Davis, J. M. (1997). The Altman Self-Rating Mania Scale. Biological Psychiatry, 42, 948-955.

American Psychiatric Association. (2013). Diagnostic and statistical manual of mental disorders $\left(5^{\text {th }}\right.$ ed.). Washington, DC: Author.

Carpenter, R. W., Zimmerman, M., Emery, N. N., \& Stanton, K. (2020). Associations between the Mood Disorder Questionnaire and psychopathology in a clinical sample. Assessment. Advance online publication.

Chmielewski, M., \& Watson, D. (2009). What is being assessed and why it matters: The impact of transient error on trait research. Journal of Personality and Social Psychology, 97, 186202.

Cohen, J. (1988). Statistical power analysis for the behavioral sciences (2nd ed.). Hillsdale, NJ: Lawrence Erlbaum Associates.

Cyders, M. A., Smith, G. T., Spillane, N. S., Fischer, S., Annus, A. M., \& Peterson, C. (2007). Integration of impulsivity and positive mood to predict risky behavior: Development and validation of a measure of positive urgency. Psychological Assessment, 19, 107-118.

Depue, R. A., Krauss, S., Spoont, M. R., \& Arbisi, P. (1989). General Behavior Inventory identification of unipolar and bipolar affective conditions in a nonclinical university population. Journal of Abnormal Psychology, 98, 117-126.

Eckblad, M., \& Chapman, L. J. (1986). Development and validation of a scale for hypomanic personality. Journal of Abnormal Psychology, 95, 214-222.

Gnambs, T. (2014). A meta-analysis of dependability coefficients (test-retest reliabilities) for measures of the Big Five. Journal of Research in Personality, 52, 20-28. 
Hirschfeld, R. M. A., Williams, J. B. W., Spitzer, R. L., Calabrese, J. R., Flynn, L., Keck, P. E., ... Zajecka, J. (2000). Development and validation of a screening instrument for bipolar spectrum disorder: The Mood Disorder Questionnaire. The American Journal of Psychiatry, $157,1873-1875$.

John, O. P., \& Srivastava, S. (1999). The Big Five trait taxonomy: History, measurement, and theoretical perspectives. In L. A. Pervin \& O. P. John (Eds.), Handbook of personality (2nd ed., pp. 102-138). New York: Guilford.

Krueger, R. F., Derringer, J., Markon, K. E., Watson, D., \& Skodol, A. E. (2012). Initial construction of a maladaptive personality trait model and inventory for $D S M-5$. Psychological Medicine, 42, 1879-1890.

Miller, C. J., Johnson, S. L., \& Eisner, L. (2009). Assessment tools for adult bipolar disorder. Clinical Psychology: Science and Practice, 16, 188-201.

Mohammadzadeh, A., \& Monazzami, M. (2018). Validation of the Hypomanic Personality Scale (HPS) in Iranian adolescents. Personality and Mental Health, 13, 15-23.

Robinson, M. D., \& Clore, G. L. (2002). Belief and feeling: Evidence for an accessibility model of emotional self-report. Psychological Bulletin, 128, 934-960.

Ruggero, C. J., Kotov, R., Watson, D., Kilmer, J. N., Perlman, G., \& Liu, K. (2014). Beyond a single index of mania symptoms: Structure and validity of subdimensions. Journal of Affective Disorders, 161, 8-15.

Schalet, B. D., Durbin, C. E., \& Revelle, W. (2011). Multidimensional structure of the Hypomanic Personality Scale. Psychological Assessment, 23, 504-522. 
Sperry, S. H., Barrantes-Vidal, N. \& Kwapil, T. R. (2018). The association of affective temperaments and bipolar spectrum psychopathology: An experience sampling study. Motivation and Emotion, 42, 126-136.

Sperry, S.H., Walsh, M.A., Kwapil, T.R. (2015). Measuring the validity and psychometric properties of a short form of the Hypomanic Personality Scale. Personality and Individual Differences, 82, 52-57.

Stanton, K., Gruber, J. \& Watson, D. (2017). Basic dimensions defining mania risk: A structural approach. Psychological Assessment, 29, 304-319.

Stanton, K., Khoo, S., Watson, D., Gruber, J., Zimmerman, M., \& Weinstock, L. M. (2019). Unique and transdiagnostic symptoms of hypomania/mania and unipolar depression. Clinical Psychological Science, 7, 471-487.

Stanton, K., McDonnell, C. G., Hayden, E. P., \& Watson, D. (2020). Transdiagnostic approaches to psychopathology measurement: Recommendations for measure selection, data analysis, and participant recruitment. Journal of Abnormal Psychology, 129, 21-28.

Stanton, K., \& Watson, D. (2017). Explicating the structure and relations of the Mood Disorder Questionnaire: Implications for screening for bipolar and related disorders. Journal of Affective Disorders, 220, 72-78.

Watson, D. (2004). Stability versus change, dependability versus error: Issues in the assessment of personality over time. Journal of Research in Personality, 38, 319-350.

Watson, D., Clark, L. A., \& Tellegen, A. (1988). Development and validation of brief measures of positive and negative affect: The PANAS scales. Journal of Personality and Social Psychology, 54, 1063-1070. 
Watson, D., \& Naragon-Gainey, K. (2010). On the specificity of positive emotional dysfunction in psychopathology: Evidence from the mood and anxiety disorders and schizophrenia/schizotypy. Clinical Psychology Review, 30, 839-848.

Watson, D., \& Naragon-Gainey, K. (2014). Personality, emotions, and the emotional disorders. Clinical Psychological Science, 2, 422-442.

Watson, D., O’Hara, M. W., Naragon-Gainey, K., Koffel, E., Chmielewski, M., Kotov, R., .. . Ruggero, C. J. (2012). Development and validation of new anxiety and bipolar symptom scales for an expanded version of the IDAS (the IDAS-II). Assessment, 19, 399-420. 
Table 1

Descriptive Statistics

Time 1

Time 2

\begin{tabular}{lrrrrrrr}
\hline Scale & M & SD & Alpha & M & SD & Alpha & $d$ \\
\hline HPS Social Vitality (19) & 6.44 & 4.00 & .79 & 6.54 & 4.04 & .79 & -.03 \\
HPS Mood Volatility (13) & 4.46 & 3.08 & .77 & 4.11 & 3.13 & .79 & .07 \\
HPS Excitement (7) & 1.70 & 1.90 & .76 & 1.67 & 1.82 & .74 & .01 \\
GBI Mania (21) & 33.53 & 9.37 & .91 & 31.74 & 9.88 & .94 & .10 \\
GBI Biphasic (7) & 11.94 & 3.65 & .80 & 11.27 & 3.63 & .83 & .12 \\
PID-5 Lability (7) & 13.62 & 5.43 & .93 & 12.74 & 5.29 & .93 & .12 \\
MDQ (13) & 6.31 & 3.69 & .85 & 5.22 & 3.69 & .85 & $.26 *$ \\
ASRM (5) & 9.10 & 3.08 & .72 & 8.93 & 3.20 & .76 & .08 \\
IDAS-II Mania (5) & 8.79 & 3.97 & .83 & 8.40 & 3.99 & .87 & .07 \\
IDAS-II Euphoria (5) & 8.69 & 3.37 & .80 & 8.81 & 3.52 & .81 & -.04 \\
IDAS-II Dysphoria (10) & 21.71 & 7.76 & .89 & 19.95 & 7.24 & .89 & .16 \\
IDAS-II Lassitude (6) & 14.97 & 4.95 & .80 & 13.97 & 4.77 & .81 & .11 \\
IDAS-II Insomnia (6) & 11.62 & 4.43 & .78 & 11.21 & 4.38 & .82 & .01 \\
IDAS-II Appetite Loss (3) & 5.30 & 2.56 & .88 & 5.09 & 2.48 & .88 & .02 \\
IDAS-II Appetite Gain (3) & 6.42 & 2.83 & .77 & 6.18 & 2.51 & .73 & .05 \\
Positive Urgency (14) & 24.19 & 8.98 & .96 & 22.81 & 8.83 & .96 & .12 \\
PANAS Positive Affect (10) & 32.88 & 6.48 & .88 & 31.29 & 7.13 & .90 & $.29 *$ \\
PANAS Negative Affect (10) & 20.45 & 6.95 & .89 & 18.95 & 6.52 & .88 & .18 \\
BFI Neuroticism (8) & 23.07 & 6.03 & .83 & 22.49 & 6.00 & .84 & .04 \\
BFI Extraversion (8) & 24.58 & 6.57 & .88 & 24.89 & 6.53 & .88 & .02 \\
BFI Openness (10) & 33.52 & 6.29 & .81 & 33.02 & 6.49 & .83 & .08 \\
BFI Agreeableness (9) & 33.93 & 5.39 & .79 & 34.03 & 5.43 & .79 & .01 \\
BFI Conscientiousness (9) & 32.23 & 5.62 & .80 & 32.11 & 5.73 & .80 & .07 \\
& & & & & & & \\
\hline
\end{tabular}

Note. $N=381-382$ for Time 1 and $N=288-291$ for Time 2 . The number of items for each scale are in parentheses following the scale name. Cohen's $d$ values that are starred (*) indicate a small effect size, and values that are italicized indicate a significant difference between Times 1 and 2 . When computing Cohen's $d$ s and conducting significance tests, we used data only from participants who completed both assessments; thus, the means and standard deviations in these analyses differed slightly from those listed above for Time 1. HPS = Hypomanic Personality Scale. GBI= General Behavior Inventory. PID-5= Personality Inventory for DSM-5. MDQ= Mood Disorder Questionnaire. ASRM= Altman Self-Rating Mania Scale. IDAS-II = Expanded Version of the Inventory of Depression and Anxiety Symptoms. PANAS $=$ Positive and Negative Affect Schedule. BFI = Big Five Inventory. 
Table 2

Correlations among the Bipolar-Relevant Measures

Scale

$\begin{array}{llllllllll}1 & 2 & 3 & 4 & 5 & 6 & 7 & 8 & 9 & 10\end{array}$

1. HPS Social

$-\quad .45$

.45

.51

$.41 \quad .28$

.32

.07

.24

$.42 \quad .30$

2. HPS Mood

$.36-$

$\begin{array}{lll}.64 & .64 & .65\end{array}$

.45

$\begin{array}{llll}.62 & .61 & .40 & .22\end{array}$

3. HPS Excitement

$.42 \quad .49$

$-$

.50

$.40 \quad .43$

$.39 \quad .48$

$.49 \quad .38$

4. GBI Mania

$.38 \quad .6$

5. GBI Biphasic

$.22 \quad .62$

$62 \quad .42$

$-\quad .85$

$85 \quad .55$

$\begin{array}{llll}.51 & .65 & .60 & .44\end{array}$

62.2

6. MDQ

$.32 \quad .56 \quad .40$

$.81-.43$

$\begin{array}{lllll}.63 & .61 & .44 & .30\end{array}$

6. $\mathrm{MDQ}$

$.32 \quad .56$

7. PID-5 Lability

8. IDAS-II Mania

$.14 \quad .6$

.64

$.53 \quad .43$

$-\quad .28$

.28

$.33 \quad .36 \quad .28$

$.20 \quad .58$

.27

$.49 \quad .61$

61.39

$\begin{array}{llll}- & .51 & .25 & .14\end{array}$

9. IDAS-II Euphoria

$.37 \quad .35$

$.58 \quad .39$

$.59 \quad .53$

$\begin{array}{lll}53 & .38 \quad .46\end{array}$

$.58 \quad .30$

10. ASRM

$.41 \quad .20 \quad .30$

$\begin{array}{lll}.51 & .36 & .36\end{array}$

$\begin{array}{llll}.21 & .50 & - & .54\end{array}$

.32

$.15 \quad .29$

$.07 \quad .22$

.49

Note. $N=381$ for Time 1 and $\mathrm{N}=288$ for Time 2 . Time 1 correlations are shown below the diagonal, and Time 2 correlations are above the diagonal. Correlations $\geq|.50|$ are bolded. Social = Social Vitality Mood $=$ Mood Volatility 
Table 3

Correlations between the Bipolar-Relevant Scales and Other Study Measures at Time 1

Scale HPS Soc HPS Mood HPS Excite GBI Mania GBI Biph MDQ PID Labil Mania Euphoria ASRM

\begin{tabular}{|c|c|c|c|c|c|c|c|c|c|c|}
\hline \multicolumn{11}{|l|}{ Depression } \\
\hline Dypshoria & .06 & .53 & .18 & .52 & .62 & .30 & .56 & .61 & .22 & .01 \\
\hline Lassitude & .02 & .40 & .13 & .42 & .49 & .29 & .39 & .42 & .23 & .05 \\
\hline Insomnia & .13 & .33 & .10 & .45 & .39 & .26 & .25 & .42 & .24 & .17 \\
\hline Appetite Gain & .08 & .27 & .22 & .33 & .33 & .23 & .28 & .42 & .33 & .19 \\
\hline Appetite Loss & .04 & .24 & .06 & .32 & .37 & .15 & .20 & .31 & .19 & .07 \\
\hline \multicolumn{11}{|c|}{ Affectivity/Personality } \\
\hline Negative Affect & .11 & .51 & .25 & .50 & .54 & .33 & .57 & .50 & .27 & .11 \\
\hline Neuroticism & -.17 & .44 & .05 & .25 & .47 & .16 & .63 & .32 & -.07 & -.16 \\
\hline Positive Urgency & .27 & .48 & .31 & .52 & .44 & .37 & .40 & .36 & .33 & .26 \\
\hline Extraversion & .60 & .06 & .39 & .07 & -.07 & .12 & -.04 & .02 & .20 & .29 \\
\hline Positive Affect & .30 & -.06 & .22 & .09 & -.05 & .13 & -.18 & .06 & .31 & .30 \\
\hline Agreeableness & -.14 & -.30 & -.02 & -.29 & -.31 & -.24 & -.25 & -.17 & -.11 & -.02 \\
\hline Conscientiousness & -.13 & -.34 & -.23 & -.27 & -.22 & -.20 & -.30 & -.17 & -.15 & -.03 \\
\hline Openness & .39 & .18 & .06 & .12 & .13 & .10 & .05 & .05 & .08 & .15 \\
\hline
\end{tabular}

Note. $\quad N=381$. Correlations $\geq|.40|$ are bolded. Soc $=$ Social Vitality; Mood = Mood Volatility; Excite $=$ Excitement. 
Table 4

Test-Retest Reliability Coefficients of the Bipolar-Relevant Scales

Scale $\quad$ Retest $r \quad$ Administration Instructions

$\begin{array}{lll}\text { HPS Social Vitality } & .83_{\mathrm{a}} & \text { In general } \\ \text { PID-5 Emotional Lability } & .77_{\mathrm{ab}} & \text { In general } \\ \text { GBI Biphasic } & .72_{\mathrm{bc}} & \text { Frequency } \\ \text { HPS Excitement } & .72_{\mathrm{bc}} & \text { In general } \\ \text { HPS Mood Volatility } & .71_{\mathrm{bc}} & \text { In general } \\ \text { GBI Mania } & .70_{\mathrm{bc}} & \text { Frequency } \\ \text { Mood Disorder Questionnaire } & .68_{\mathrm{cd}} & \text { Ever occurred } \\ \text { IDAS-II Mania } & .64_{\mathrm{cd}} & \text { Past } 2 \text { weeks } \\ \text { IDAS-II Euphoria } & .60_{\mathrm{de}} & \text { Past } 2 \text { weeks } \\ \text { ASRM } & .49_{\mathrm{e}} & \text { Past week }\end{array}$

Note. $N=287$. Coefficients with the same subscript did not differ significantly from one another. 
Table 5

Test-Retest Reliability Coefficients of the Personality, Affect, and Psychopathology Scales

Scale $\quad$ Retest $r \quad$ Administration Instructions

\begin{tabular}{|c|c|c|}
\hline BFI Extraversion & $.90 \mathrm{a}$ & In general \\
\hline BFI Openness & $.85_{\mathrm{b}}$ & In general \\
\hline BFI Neuroticism & $.85_{\mathrm{b}}$ & In general \\
\hline BFI Conscientiousness & $.84_{b}$ & In general \\
\hline BFI Agreeableness & $.83_{\mathrm{b}}$ & In general \\
\hline IDAS-II Dysphoria & $.74_{\mathrm{c}}$ & Past 2 weeks \\
\hline PANAS Positive Affect & $.73_{\mathrm{c}}$ & In general \\
\hline PANAS Negative Affect & $.73_{\mathrm{c}}$ & In general \\
\hline Positive Urgency Measure & $.67_{\mathrm{cd}}$ & In general \\
\hline IDAS-II Lassitude & $.66_{\mathrm{cd}}$ & Past 2 weeks \\
\hline IDAS-II Appetite Gain & $.63_{\mathrm{de}}$ & Past 2 weeks \\
\hline IDAS-II Insomnia & $.61_{\mathrm{de}}$ & Past 2 weeks \\
\hline IDAS-II Appetite Loss & $.566_{\mathrm{e}}$ & Past 2 weeks \\
\hline
\end{tabular}

Note. $N=286$. Coefficients with the same subscript did not differ significantly from one another. 